\title{
A geometry constraint handling technique for stiffener layout optimization problem
}

\author{
Joon-Ho Lee, Gyeong-Ho Kim, Youn-Sik Park* \\ Center for Noise and Vibration Control (NOVIC), Department of Mechanical Engineering, Korea Advanced Institute of \\ Science and Technology (KAIST), Science Town, Daejeon 305-701, Republic of Korea
}

Received 11 March 2004; received in revised form 5 August 2004; accepted 16 August 2004

Available online 23 November 2004

\begin{abstract}
Beam stiffeners have frequently been used for raising natural frequencies of base structures. In stiffener layout optimization problems, most of the previous researches considering the position and/or the length of the stiffener as design variables dealt with structures having just simple convex shapes such as a square or rectangle. The reason was because concave shape structures have difficulties in formulating geometry constraints. In this paper, a new geometry constraint handling technique, which can define both convex and concave feasible regions and measure a degree of geometry constraint violation, was proposed. Evolution strategies (ESs) was utilized as an optimization tool. In addition, the constraint-handling technique of EVOSLINOC (EVOlution Strategies for scalar optimization with LInear and NOnlinear Constraints) was utilized to solve constrained optimization problems. From numerical examples, the proposed geometry constraint handling technique was verified and proved that the technique can easily be applied to structures in not only convex but also concave shapes, even with a protrusion or interior holes.
\end{abstract}

(C) 2004 Elsevier Ltd. All rights reserved.

\section{Introduction}

Structural dynamics modification (SDM) technique is widely used to optimally modify a base structure by adding or deleting auxiliary (modifying) structures to improve dynamic characteristics, e.g. natural frequency, mode shape and frequency response function (FRF), of

\footnotetext{
${ }^{*}$ Corresponding author. Tel.: + 82428693020 ; fax: +82428698220 .

E-mail addresses: justinlee@kaist.ac.kr (J.-H. Lee), kimgyeongho@kaist.ac.kr (G.-H. Kim), yspark0117@kaist.ac.kr (Y.-S. Park).
} 
the base structure. There have been hundreds of research papers on SDM since the late 1970s. The overall methodology of the technique and issues (e.g. mode truncation, lack of rotational dof, etc.) were summarized by Avitabile [1].

Beam stiffeners have frequently been used for raising natural frequencies of base structures. Adding stiffeners increases small weight, but its influence on the total stiffness is enormous. Traditionally, the cross-sectional dimensions of stiffeners are considered as main design variables while their positions or lengths are predetermined in the optimization process [2]. However, it was claimed that the overlooked variables (i.e. positions and lengths of stiffeners) also affect the dynamics of base structures significantly [3-9].

If the positions as well as the lengths of stiffeners are considered as design variables and stiffeners are geometrically constrained to be placed within the base structure (i.e. feasible stiffener positioning region), a stiffener layout optimization problem becomes a constrained optimization one. In case of a convex feasible region, the feasible region can be mathematically described with inequality constraints. However, in case of a concave feasible region, it is difficult to describe the feasible region in mathematical forms. In order to handle the second case easily and efficiently, geometry algorithms [10,11], which are used in the fields of computer graphics and imaging, computer vision and recognition, etc., are adopted in this paper.

Based on the feasible region defined by geometry algorithms, evolution strategies (ESs) [12-14], which is a probabilistic population-based optimization technique, is used. The technique finds an optimal solution by processing a population of design points among the space of variables based on the principles from biological evolution in nature. In addition, the constraint-handling technique of EVOSLINOC (EVOlution Strategies for scalar optimization with LInear and NOnlinear Constraints), proposed by Binh and Korn [15], is utilized to solve constrained optimization problems using ESs.

The objectives of this paper are: (1) to propose a geometry constraint-handling technique, which can define both convex and concave feasible stiffener positioning regions and measure a degree of geometry constraint violation; (2) to apply the technique to stiffener layout optimization problem to raise the first natural frequency of the base structure.

The remainder of the paper is organized as follows. In Section 2, a target problem, i.e. stiffener layout optimization, is defined first and then, the necessity of geometry algorithms in stiffener layout optimization is described. Section 3 explains how to represent a feasible region and to perform a feasibility check by geometry algorithms. Section 4 describes the EVOSLINOC, which is used to solve constrained optimization problems. In Section 5, a special strategy to measure a degree of geometry constraint violation is proposed. In Section 6, numerical examples of stiffener layout optimization to raise the first natural frequency of an L-shaped plate structure and a front panel of air conditioner outdoor unit are presented to verify the proposed technique.

\section{Stiffener layout optimization}

\subsection{Target problem definition}

The goal of this paper is to optimize stiffener layout to raise the first natural frequency $\omega_{1}$ of a base plate structure, as explained in Fig. 1. The design variables $\mathbf{x}$ include the coordinates of one 
end of stiffener $\left(x_{i}, y_{i}\right)$, stiffener rotation angle $\theta_{i}$, and its length $L_{i}$. Assuming that the stiffener is geometrically constrained to be fully placed within the base plate, which is called a geometry constraint in this paper, the target problem becomes a constrained optimization one.

\subsection{Necessity of geometry algorithms in stiffener layout optimization}

If a base plate has a convex (rectangular in this case) shape as in Fig. 2, the geometry constraint can be mathematically described with inequality constraints as

$$
\begin{aligned}
& 0 \leqslant x_{i}, x_{i}+L_{i} \cos \theta_{i} \leqslant a, \\
& 0 \leqslant y_{i}, y_{i}+L_{i} \sin \theta_{i} \leqslant b .
\end{aligned}
$$

The constraint formulas should be provided by an engineer when solving the constrained optimization problem. Note that a point $\mathbf{x}$ that satisfies Eq. (1) is said to be feasible, where $\mathbf{x}=\left(x_{i}, y_{i}, L_{i}, \theta_{i}\right)$.

In general, the stiffener is modeled with beam elements and its geometry is represented by a line segment. Thus, Eq. (1) corresponds to the condition that a line segment is included in a 2D planar convex polygon. Note that a planar polygon is convex if it contains all the line segments connecting any pair of its points. However, in case a base plate has a concave shape as Fig. 3, it is difficult to describe the geometry constraint mathematically with just the coordinates of two end points of the stiffener, which is the main reason why most of the previous researches [3-9] dealt with just simple convex shapes such as a square or rectangle. If there are void spaces or obstacles
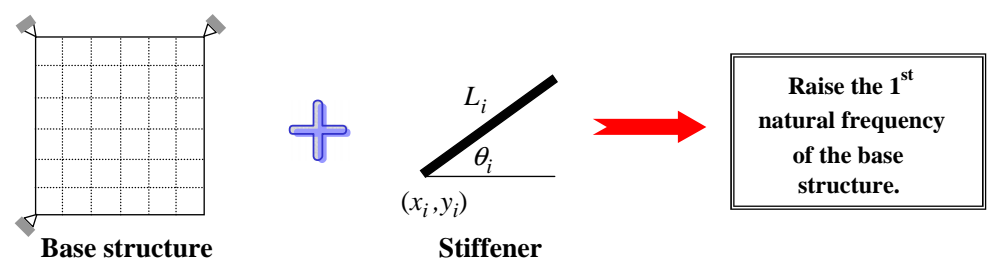

Stiffener

Fig. 1. Target problem to be considered.

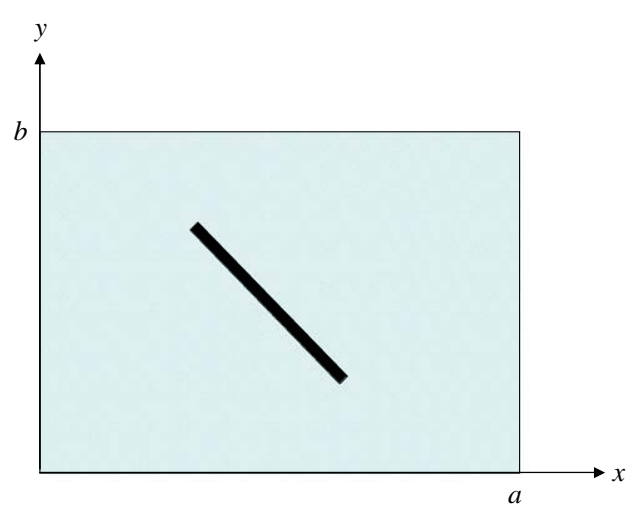

Fig. 2. Convex feasible region. 


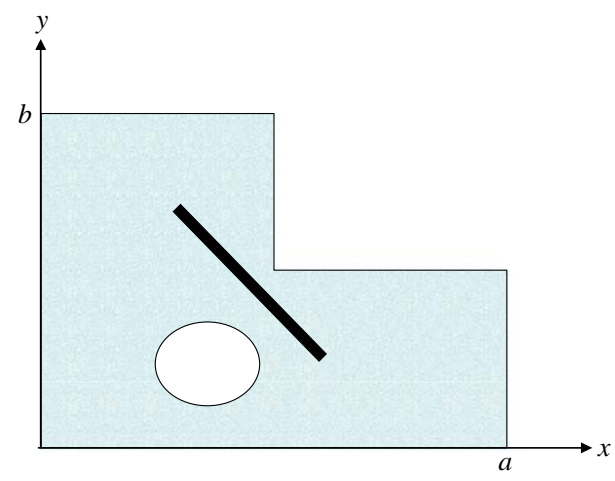

Fig. 3. Concave feasible region.

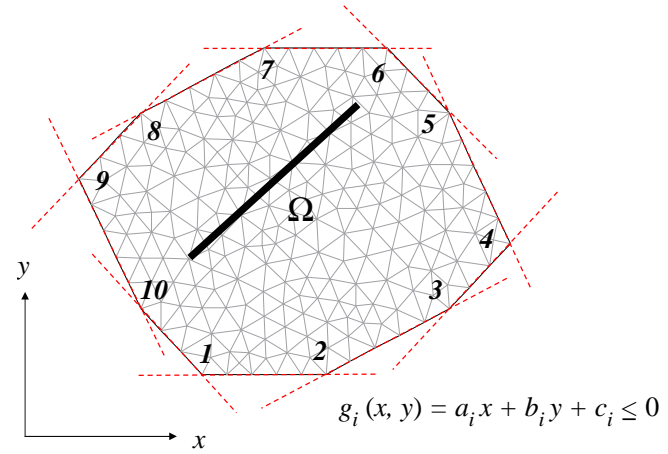

Fig. 4. A complex region which has ten inequality constraints to define a region $\Omega$.

inside the base plate, where the stiffener cannot be placed, it becomes more difficult. In addition, if the base plate shape is complex, many inequality constraints are needed to define a feasible region $\Omega$. For example, Fig. 4 shows ten vertexes to define a region $\Omega$ of FE model of base plate. Thus, twenty inequality constraint formulas should be constructed, i.e. ten inequality constraint formulas $\left(g_{i}(x, y)=a_{i} x+b_{i} y+c_{i} \leqslant 0, i=1, \ldots, 10\right)$ for each end of stiffener. As the number of vertexes to define a feasible region increases, the more inequality constraint formulas are needed. However, if geometry algorithms are utilized, not only the description of both convex and concave feasible regions but also the feasibility check of design variables $\mathbf{x}$ can be performed easily and efficiently. Note that the only necessary thing is the information of coordinates of polygon vertexes which are used to define a feasible region.

\section{Description of a feasible region and feasibility check by geometry algorithms}

\subsection{Inclusion of a point in a polygon}

The constraint that a stiffener should be fully placed within the base plate can be stated as $a$ line segment should be included in a 2 D planar polygon, where the stiffener is represented by a line 


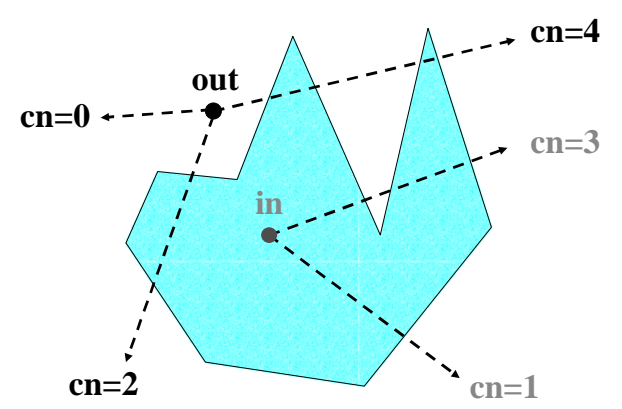

Fig. 5. Test for inclusion of a point in a planar polygon (Ref. [11]).

segment while the domain of the base plate (i.e. feasible stiffener positioning region) is represented by a $2 \mathrm{D}$ planar polygon. Because a line segment is defined by two end points, a test for inclusion of a point in a 2D planar polygon should be performed first.

Fig. 5 shows the crossing number method [11], which counts the number of times a ray starting from the point crosses the polygon boundary edges. The point is outside when this crossing number is even; otherwise, when it is odd, the point is inside. The crossing number method can be applied to not only convex but also concave polygons.

\subsection{Inclusion of a line segment in a polygon}

In case of convex polygons, as shown in Fig. 6, a line segment is included in a polygon only if two end points of the line segment are included in the polygon. However, in case of concave polygons, an additional test is needed: a test for crossing between the line segment and the polygon boundary edges, as shown in Fig. 7. Thus, in case of concave polygons, a line segment is included in a polygon if (1) two end points of the line segment are included in the polygon and (2) there is no crossing between the line segment and the polygon boundary edges.

\subsection{Inclusion of a polygon in a polygon (supplementary)}

Based on the test for inclusion of a line segment in a polygon, the inclusion of a polygon in a polygon can also be tested. Assume that the feasible region is defined by intersection of two regions: (1) region inside a polygon $\mathbf{V} \mathbf{1}_{\text {out }}$; (2) region outside of a polygon $\mathbf{V} \mathbf{1}_{\text {in }}$, as shown in Fig. 8. Thus, a polygon $\mathbf{V} \mathbf{2}$ is in the feasible region if the following requirements are satisfied:

(1) All of the edges of the polygon $\mathbf{V} \mathbf{2}$ should be inside the polygon $\mathbf{V} \mathbf{1}_{\text {out }}$.

(2) All of the edges of the polygon $\mathbf{V} \mathbf{2}$ should be outside of the polygon $\mathbf{V} \mathbf{1}_{\text {in }}$.

(3) All of the edges of the polygon $\mathbf{V} \mathbf{1}_{\text {in }}$ should not be included in the polygon $\mathbf{V} \mathbf{2}$.

Note that the edges of a polygon are line segments themselves and the polygon $\mathbf{V} \mathbf{1}_{\text {in }}$ represents, for example, a protrusion or interior holes.

In summary, a feasible region is defined by coordinates of vertexes of a planar polygon, which can easily be obtained from FE model of the base plate, and a feasibility check can easily be performed by a test for inclusion of a line segment in a planar polygon. Note that these methods 


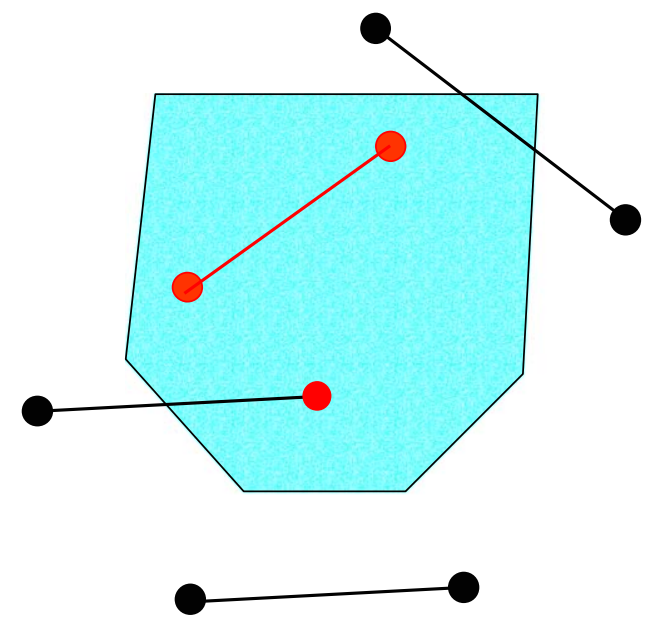

Fig. 6. Test for inclusion of a line segment in a convex polygon.

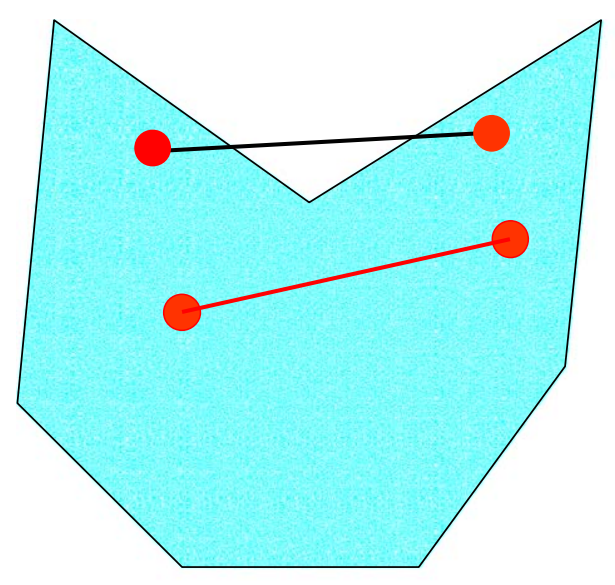

Fig. 7. Test for inclusion of a line segment in a concave polygon.

can also be applied to a $3 \mathrm{D}$ planar polygon with the surface normal vector $\mathbf{n}=\left(n_{x}, n_{y}, n_{z}\right)$ by transformation of coordinates from $\mathbf{n}=\left(n_{x}, n_{y}, n_{z}\right)$ to $\hat{\mathbf{n}}=(0,0,1)$.

\section{EVOSLINOC}

In order to handle geometry constraints by geometry algorithms, it is impossible to use gradient-based optimization techniques, e.g. steepest descent method, conjugate gradient method, quasi-Newton method, etc., as an optimization tool because geometry constraints, defined by 


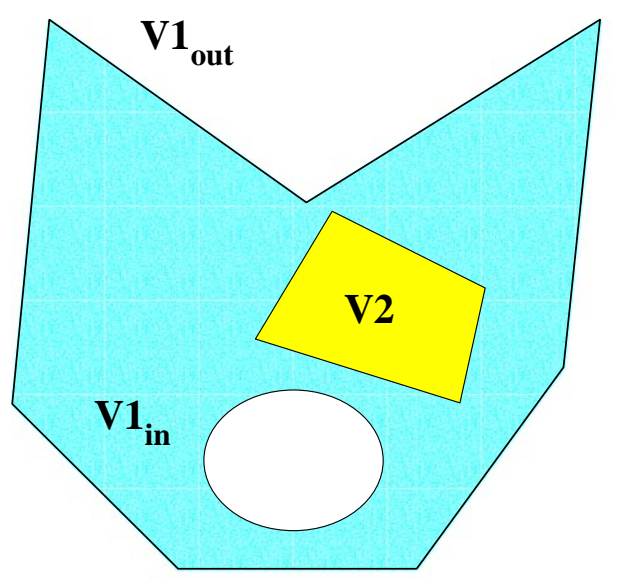

Fig. 8. Test for inclusion of a polygon in a polygon.

geometry algorithms, are not differentiable. Therefore, ESs [12-14], which is one of nongradientbased optimization techniques, is utilized in this paper.

ESs is a probabilistic population-based optimization method that mimics the principle from biological evolution in nature. In the ESs, each population is composed of design variables and strategy parameters for mutation size. ESs is largely classified into two types of $(\mu, \lambda)$-ESs and $(\mu+\lambda)$-ESs. In the $(\mu, \lambda)$-ESs, $\mu$ parents produce $\lambda(>\mu \geqslant 1)$ offspring by recombination and mutation at every generation and then, the $\mu$ best offspring are selected deterministically from the $\lambda$ offspring and replace the current parents. As a result, the lifetime of an elite individual is one generation. In the $(\mu+\lambda)$-ESs, on the other hand, $\mu$ parents produce $\lambda$ offspring by recombination and mutation at every generation and both parents and offspring compete to be the $\lambda$ parents of the next generation. The details of ESs such as procedures of mutation and recombination can be found in Refs. [12-14].

Evolutionary algorithms (EAs), whose three main paradigms are genetic algorithms (GA), evolutionary programming (EP) and ESs, are inherently unconstrained optimization procedures. Therefore, it is necessary to find ways of incorporating the constraints into the objective function in order to solve constrained optimization problems by using EAs [16]. The most common approach to handle constraints is based on the concept of penalty functions, where the constrained optimization is converted into the unconstrained optimization of an auxiliary scalar function $\phi(\mathbf{x})$ which is created from the given objective function $f(\mathbf{x})$ and the amount of constraint violation present in a certain solution $\mathbf{x}$, for example, as Eq. (2)

$$
\phi(\mathbf{x})=f(\mathbf{x}) \pm\left[\sum_{i=1}^{p} r_{i} \cdot \max \left\{g_{i}(\mathbf{x}), 0\right\}^{\beta}+\sum_{j=1}^{m} s_{j} \cdot\left|h_{j}(\mathbf{x})\right|^{\gamma}\right] \quad(\beta, \gamma=1 \text { or } 2)
$$

where $g_{i}(\mathbf{x})$ and $h_{j}(\mathbf{x})$ are inequality and equality constraints, respectively. $r_{i}$ and $s_{j}$ are weighting factors. The basic problem of all penalty approaches, however, is how to design an auxiliary scalar function $\phi(\mathbf{x})$. Therefore, their performances are problem-dependent. 
Traditional ESs uses death penalty method that eliminates infeasible individuals from the population. Thus, ESs cannot start until a feasible initial population is generated. In addition, it is not easy to find feasible individuals especially in case the feasible region is very narrow.

To cope with these drawbacks, Binh and Korn [15] proposed a new ESs for scalar optimization with linear and nonlinear constraints (EVOSLINOC). The EVOSLINOC has the following characteristics:

- It is not necessary to provide a feasible initial population.

- The handling of the objective function and constraints is performed separately. Therefore, no auxiliary function is needed.

- Infeasible individuals are allowed during the optimization.

In EVOSLINOC, an individual is represented as follows:

$$
\mathrm{Ind} \triangleq(\mathbf{x}, \mathbf{s}, f(\mathbf{x}), C(\mathbf{x}))
$$

where $\mathbf{x}$ is a vector of design variables and $\mathbf{s}$ is a strategy parameter vector. $f(\mathbf{x})$ is an objective function value ( $F$-fitness) and $C(\mathbf{x})$ is a degree of violation of constraints or degree of infeasibility ( $C$-fitness). In general, $C$-fitness of an individual is defined by

$$
C(\mathbf{x})=\left(\sum_{i=1}^{p}\left[c_{i}(\mathbf{x})\right]^{r}\right)^{1 / r}, \quad(r>0)
$$

where $c_{i}(\mathbf{x})=\max \left\{g_{i}(\mathbf{x}), 0\right\}, i=1, \ldots, p$.

Different to traditional ESs, both feasible and infeasible individuals can live in the population simultaneously in EVOSLINOC. The reason why infeasible individuals should be alive in the population can be explained in Fig. 9. An infeasible individual ' $b$ ' lies nearer to the feasible global minimum than a feasible one ' $a$ '. For the same strategy parameter vector $\mathbf{s}$, it is expected that the infeasible individual ' $b$ ' generates feasible offspring better than offspring of the feasible individual ' $a$ '. Thus, keeping such infeasible individuals in the population increases the possibility of finding the global minimum. In addition, niche (in)feasible individuals are used in EVOSLINOC because they play an important role to overcome local minima in multi-modal optimization problems. In Fig. 10, an

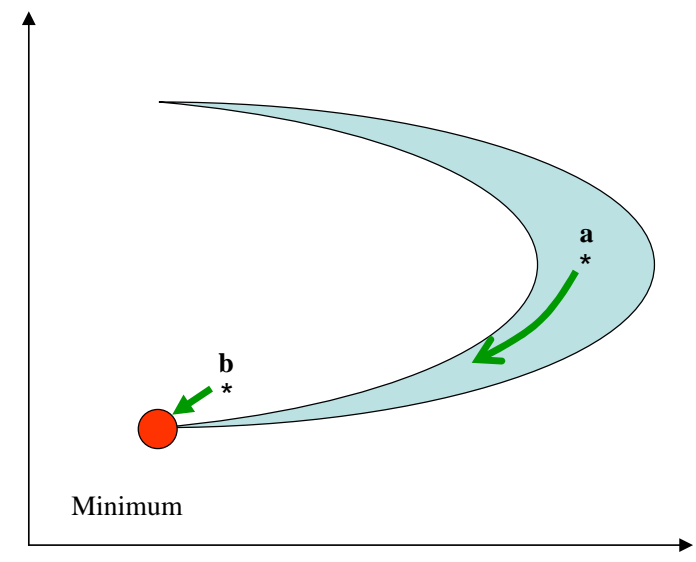

Fig. 9. A feasible individual 'a' and an infeasible individual 'b' (Ref. [15]). 


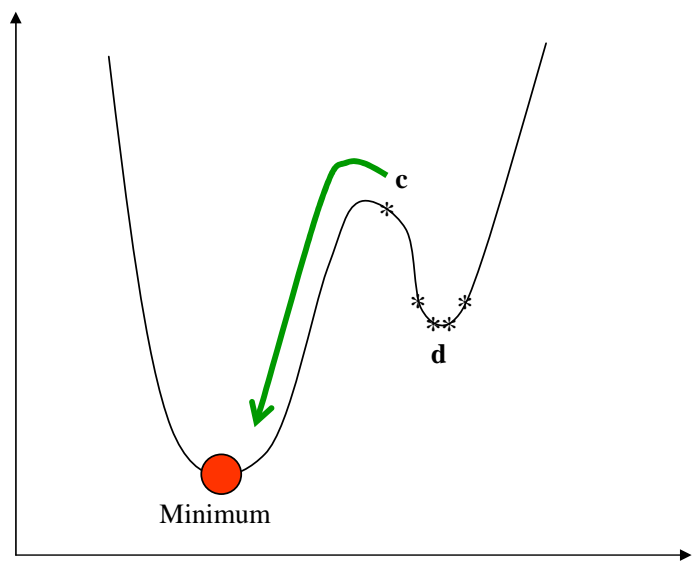

Fig. 10. Niche individual 'c'.

individual ' $c$ ' is not better than other individuals ' $d$ ' that are stuck in a local minimum. However, it is expected that the individual ' $c$ ' generates offspring better than offspring of other individuals ' $d$ ' for finding the global minimum. Global selection scheme to create parents for the next generation from the current population, which is a key point in the constraint-handling, is summarized as follows:

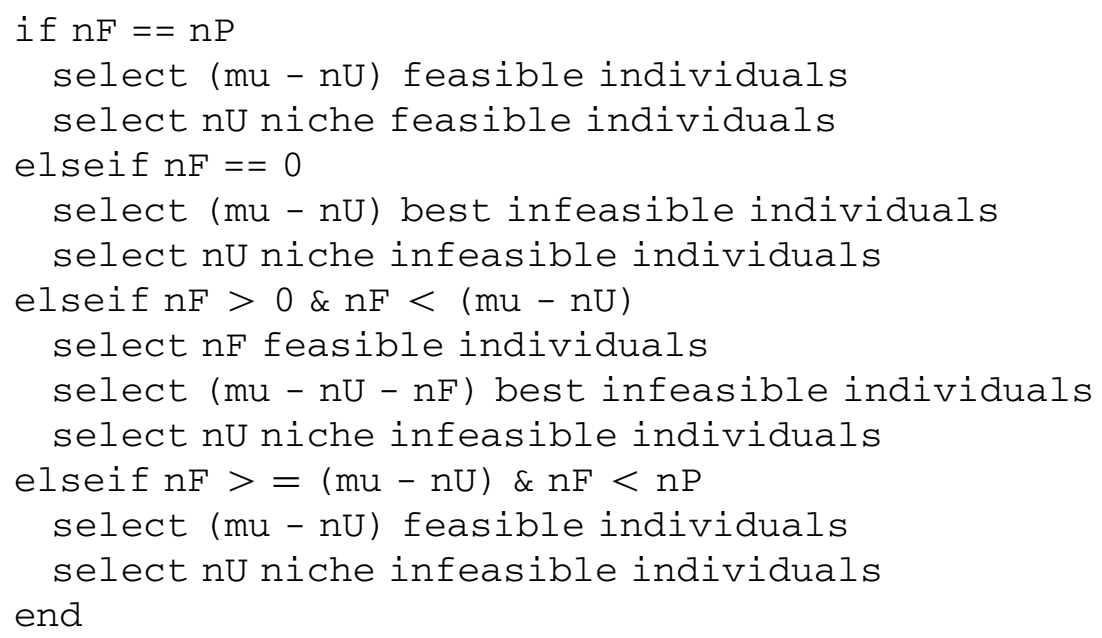

where $\mathrm{nP}, \mathrm{nF}, \mathrm{nU}$ and $\mathrm{mu}$ are the population size, the number of feasible individuals of the current population, the number of niche (in)feasible individuals and the number of parents $\mu$, respectively. Note that $\mathrm{nU}$ can be adjusted by engineers. The details of handling feasible and infeasible individuals and niche individuals can be found in Ref. [15].

\section{Linking geometry algorithms to EVOSLINOC}

In geometry algorithms, a test for inclusion of a line segment in a planar polygon returns only two binary values: 0 for feasible or 1 for infeasible. Therefore, all of the line segments that are not 


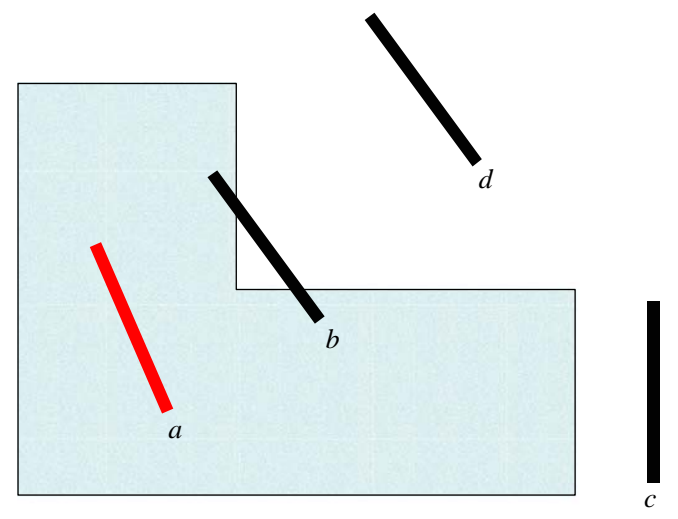

Fig. 11. A feasible individual 'a' and infeasible individuals 'b,c,d'.

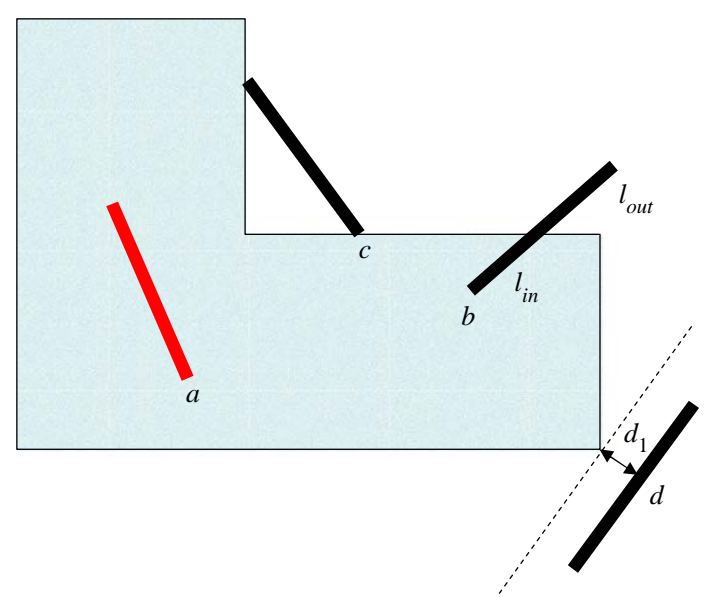

Fig. 12. Strategy to measure a degree of infeasibility.

included in a polygon come to have equal $C$-fitness values. However, as shown in Fig. 11, an infeasible line segment ' $b$ ' is better than infeasible line segments ' $c$ ' and ' $d$ ' because a fraction of the line segment ' $\mathrm{b}$ ' is inside the polygon. In addition, the infeasible line segment ' $\mathrm{c}$ ' is better than infeasible line segment ' $d$ ' because the line segment ' $c$ ' is closer to the polygon boundary edges than line segment ' $d$ '. The EVOSLINOC uses $C$-fitness value for ranking infeasible individuals. Thus, an additional strategy for ranking infeasible individuals is necessary when using geometry algorithms. Fig. 12 shows a proposed strategy to measure a degree of infeasibility, which is based on: (1) a fraction of the line segment lying outside of the polygon $\left(l_{\text {out }} /\left(l_{\text {in }}+l_{\text {out }}\right)\right)$ and (2) a distance from the line segment to the nearest polygon boundary edge if a line segment is outside of the polygon (dist). Thus, $C$-fitness value of an individual is calculated as

$$
C(\mathbf{x})=\max \left\{\frac{l_{\text {out }}}{l_{\text {in }}+l_{\text {out }}}+\text { floor }\left(\frac{l_{\text {out }}}{l_{\text {in }}+l_{\text {out }}}\right) \cdot \text { dist, } 0\right\}
$$


where $\max (x, y)$ returns the larger value between $x$ and $y$ and floor $(x)$ rounds $x$ to the nearest integer not exceeding $x$. Therefore, $C$-fitness values of four individuals in Fig. 12 are as follows:

- Segment a : 0 (feasible individual).

- Segment b: $l_{\text {out }} /\left(l_{\text {in }}+l_{\text {out }}\right)$.

- Segment c : 1 .

- Segment $\mathrm{d}: 1+d_{1}$.

Note that $F$-fitness values of four individuals become as: $-\omega_{1}(<0)$ for the feasible segment 'a' and 0 for infeasible segments ' $b$ ', 'c' and ' $d$ ', where $\omega_{1}$ is the first natural frequency of a stiffened plate. Evaluation of objective function for infeasible individuals is not necessary. It is also noted that polygon boundary edges themselves can be regarded as a portion of the feasible region at this stage.

\section{Numerical examples}

Based on the proposed geometry constraint handling technique by geometry algorithms, numerical examples of stiffener layout optimization are performed with an L-shaped plate structure and a front panel of air conditioner outdoor unit.

\subsection{L-shaped plate}

\subsubsection{Case 1}

An L-shaped base plate structure, which is simply supported at five corners as shown in Fig. 13(a), is chosen as the first example. As mentioned before, four design variables are considered: coordinates of one end of stiffener $\left(x_{i}, y_{i}\right)$, stiffener rotation angle $\theta_{i}$, and its length $L_{i}$. The plate has thickness $t_{p}=3 \mathrm{~mm}$, Young's modulus $E=200 \mathrm{GPa}$, Poisson's ratio $v=0.3$ and density $\rho=7800 \mathrm{~kg} / \mathrm{m}^{3}$. The stiffener, which is made of the same material as the plate, has cross-sectional dimensions of width $w=5 \mathrm{~mm}$ and height $h=10 \mathrm{~mm}$. First of all, a feasible stiffener positioning region is defined by a 2D planar concave polygon VO with six vertexes, as shown in Fig. 13(b). Coordinates of six vertexes are

$$
\mathbf{V O}=\left[\begin{array}{l}
\mathbf{V O} \mathbf{O}_{\mathbf{x}} \\
\mathbf{V O}_{\mathbf{y}}
\end{array}\right]=\left[\begin{array}{llllll}
0.0 & 1.5 & 1.5 & 0.5 & 0.5 & 0.0 \\
0.0 & 0.0 & 0.5 & 0.5 & 1.0 & 1.0
\end{array}\right] \quad \text { (unit: } \mathrm{m} \text { ) }
$$

The geometry of a line segment $\mathbf{S}$ representing the stiffener can be obtained from the given design variables. If a test for inclusion of the line segment $\mathbf{S}$ in the planar polygon $\mathbf{V O}$ is successful, i.e. $C(\mathbf{x})=0$, then natural frequencies of the stiffened plate are calculated [7-9,17]. Note that the displacement of the stiffener is interpolated with shape functions of the plate elements, which enables the stiffener to be arbitrarily placed on the base plate [7-9,17]. All of the techniques involved in this paper can be summarized as follows:

- Geometry algorithms $[10,11]$ to define a feasible region and to measure a degree of geometry constraint violation.

- ESs [12-14] for mutation, recombination and the overall optimization. 


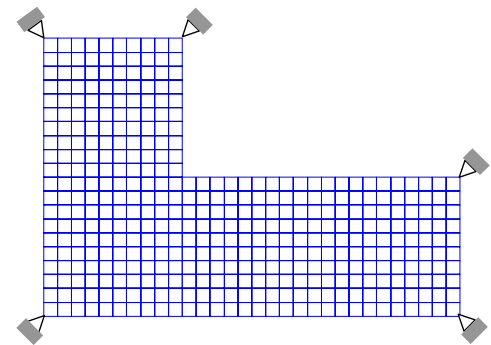

(a)

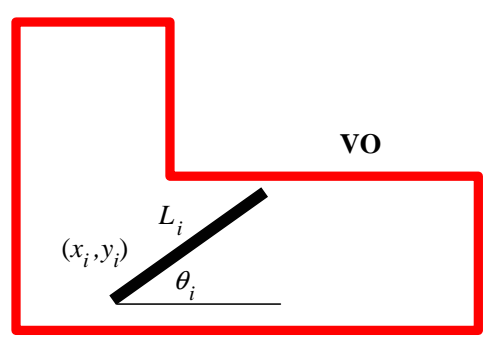

(b)

Fig. 13. (a) FE model of an L-shaped base plate, (b) a feasible region.

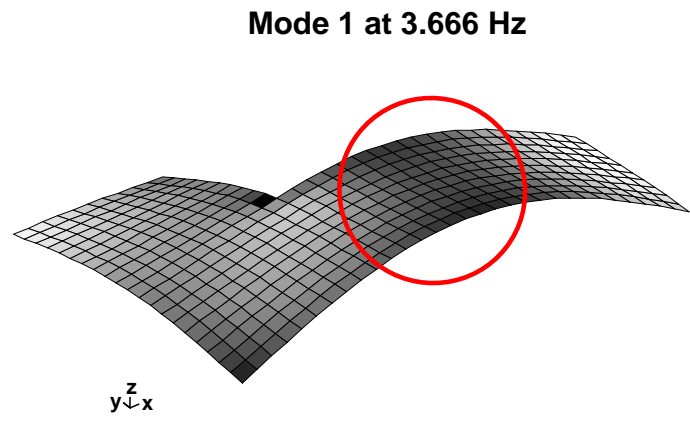

Fig. 14. Modal dynamic strain energy distribution of base plate at first mode.

- EVOSLINOC [15] for constraint-handling and global selection of the parents for the next generation.

Note that all of the necessary functions are implemented in the MATLAB environment and the MATLAB-based Structural Dynamics Toolbox [18] is used for both FE modeling and eigenvalue solving.

Fig. 14 shows modal dynamic strain energy distribution of the base plate at first mode $\left(\omega_{1}=\right.$ $3.6660 \mathrm{~Hz}$ ). First mode is a bending mode and high strain energy exists in the region marked by a circle, from which the stiffener is expected to be placed in a way to suppress the dominant vibration in that region.

In the process of ESs, the following parameters are used:

- Number of parents $\mu=40$.

- Number of offspring $\lambda=100$.

- Number of niche (in)feasible individuals $\mathrm{nU}=0.3 \times \mu=12$.

- Number of initial individuals $=40$.

- Number of population $\mathrm{nP}=\mu+\lambda=140 \Leftarrow(\mu+\lambda)$-ESs.

- Termination criterion: stop if the best value of the objective function in the last 40 generations has not been improved. 


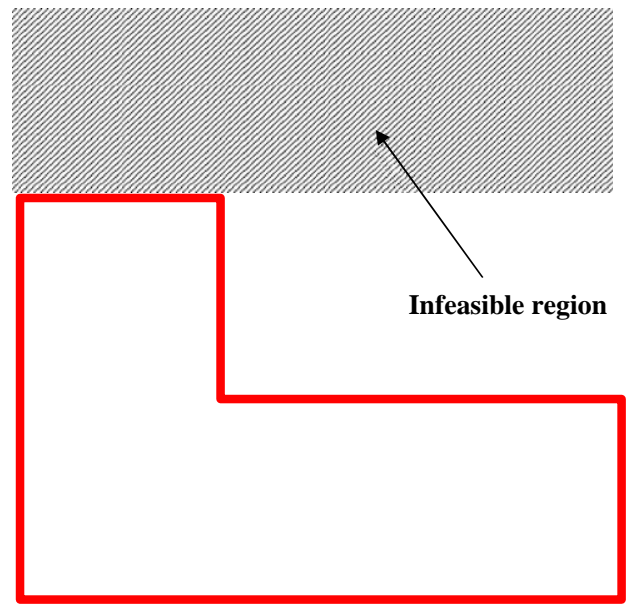

Fig. 15. Infeasible region where initial individuals are generated.

Intentionally, initial individuals with lengths less than $300 \mathrm{~mm}$ are generated randomly to be placed within the specified infeasible region, as shown in Fig. 15. Therefore, all initial individuals become infeasible.

Figs. 16 and 17 show the variation of the stiffener layout and the improvement of the first natural frequency of the stiffened plate during the optimization process, respectively. The stiffener, marked number $\mathbf{0}$, shows the elite infeasible individual among all infeasible initial individuals (i.e. individual having the lowest $C$-fitness value). Note that the elite feasible individual, which is the one having the highest first natural frequency (i.e. the lowest $F$-fitness value) among all population at a specific generation number, is plotted in Figs. 16 and 17. The obtained optimal stiffener layout is shown in Fig. 16, marked number 5. When the stiffener is placed along the bottom boundary line of the base plate, the first natural frequency $\omega_{1}$ can be increased to $3.9967 \mathrm{~Hz}$. Then the obtained feasible stiffener position is $\mathbf{x}^{*}=\left(x_{i}^{*}, y_{i}^{*}, L_{i}^{*}, \theta_{i}^{*}\right)=$ $\left(0.0000 \mathrm{~m}, 0.0000 \mathrm{~m}, 1.5000 \mathrm{~m}, 0.0000^{\circ}\right)$.

\subsubsection{Case 2}

In order to verify the performance of the proposed technique, an infeasible region is added inside the feasible region as shown in Fig. 18, which simulates the following situation: an obstacle (e.g. protrusion) exists on the base plate, which restricts the movement of the stiffener during the optimization process. Note that the added infeasible region can also represent void spaces (e.g. holes) that exist inside the base plate. The optimal stiffener layout, however, should not be changed by adding the infeasible region because the FE model of the base plate remains unchanged. The added infeasible region interferes in only the process to obtain the optimal solution. The interior infeasible region, where the stiffener cannot be placed, is defined by a $2 \mathrm{D}$ planar convex polygon VI with four vertexes. Coordinates of four vertexes are:

$$
\mathbf{V I}=\left[\begin{array}{l}
\mathbf{V I}_{\mathbf{x}} \\
\mathbf{V I}_{\mathbf{y}}
\end{array}\right]=\left[\begin{array}{llll}
0.25 & 1.25 & 1.25 & 0.25 \\
0.20 & 0.20 & 0.30 & 0.30
\end{array}\right] \text { (unit: } \mathrm{m} \text { ) }
$$




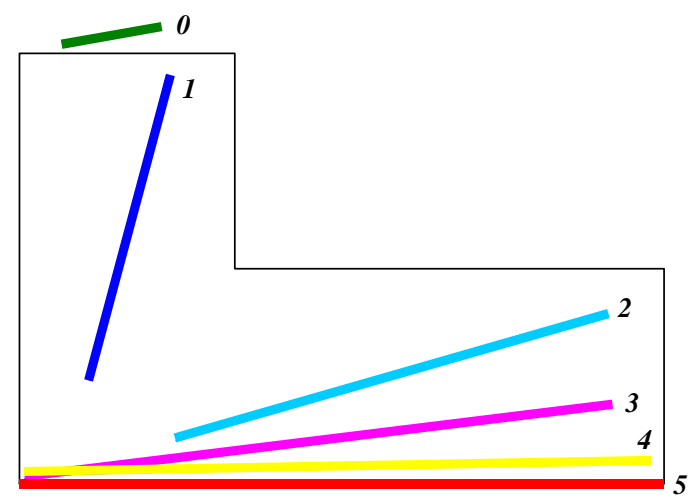

Fig. 16. Variation of stiffener layout during the optimization process (Case 1).

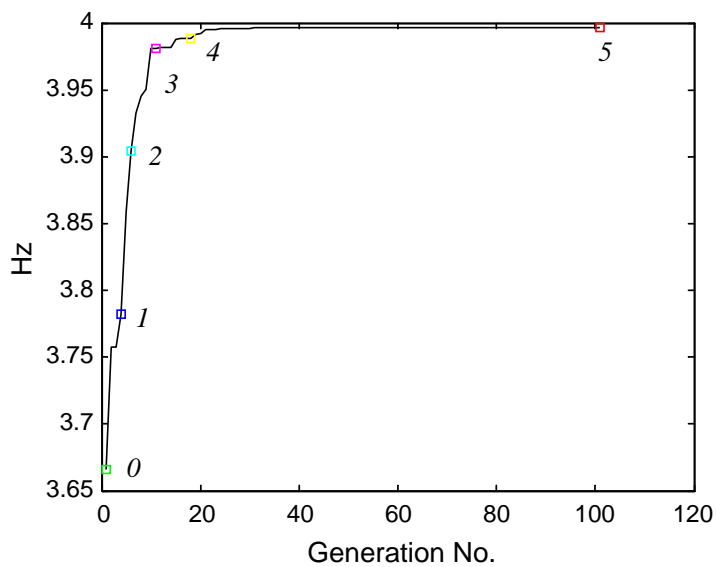

Fig. 17. Improvement of the first natural frequency of the stiffened plate (Case 1).

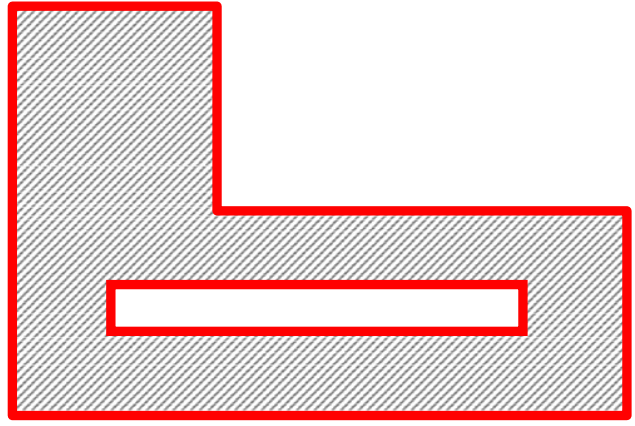

Fig. 18. Feasible region with an interior infeasible region. 
Thus, the feasible region can be defined by two planar polygons VO and VI, i.e. intersection between the region inside the polygon $\mathbf{V O}$ and the region outside of the polygon VI. Note that the feasible region is the only thing different from Case 1.

The optimal stiffener layout of Case 2, marked number $\mathbf{5}$, is shown in Fig. 19, which is nearly the same with the result of Case 1 . The obtained feasible stiffener position is $\mathbf{x}^{*}=\left(x_{i}^{*}, y_{i}^{*}, L_{i}^{*}, \theta_{i}^{*}\right)=$ $\left(0.0000 \mathrm{~m}, 0.0000 \mathrm{~m}, 1.5000 \mathrm{~m}, 360.0000^{\circ}\right)$ and the first natural frequency $\omega_{1}^{*}$ of the stiffened plate is 3.9967 Hz, as shown in Fig. 20.

\subsubsection{Case 3}

Different to Case 2, the feasible region is disjointed by an elongated interior infeasible region in Case 3, as shown in Fig. 21. Because initial individuals of Case 1, which exist above the interior infeasible region, are also used in this case, the optimizer must have an ability to cross over the interior infeasible region, below which the optimal solution exists, to get to the optimal solution.

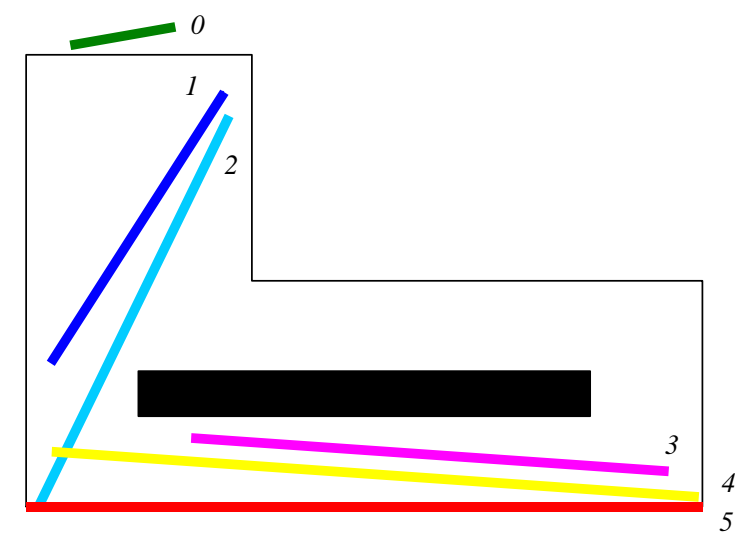

Fig. 19. Variation of stiffener layout during the optimization process (Case 2).

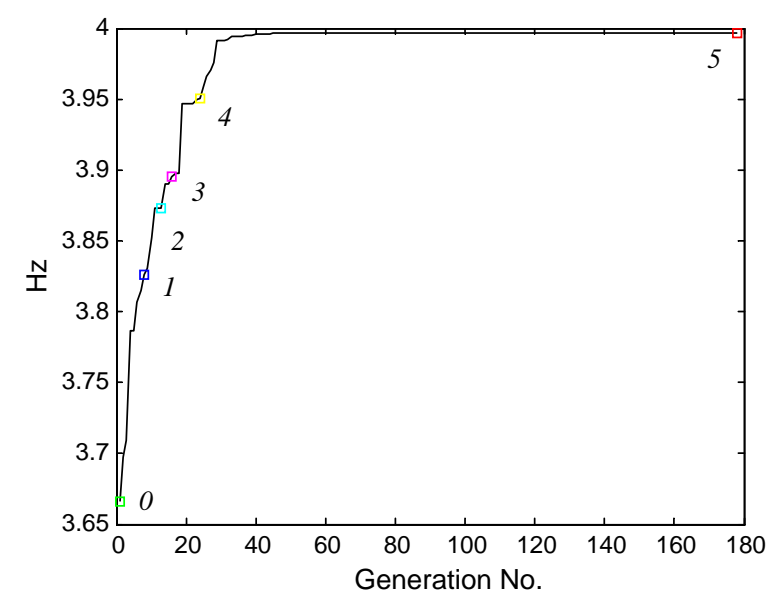

Fig. 20. Improvement of the first natural frequency of the stiffened plate (Case 2). 


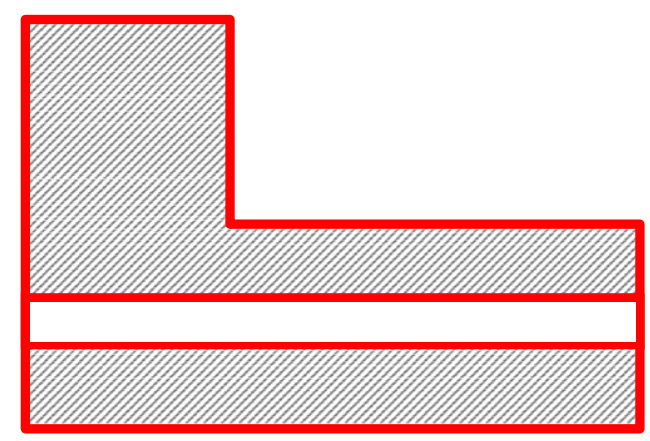

Fig. 21. Feasible region disjointed by an interior infeasible region.

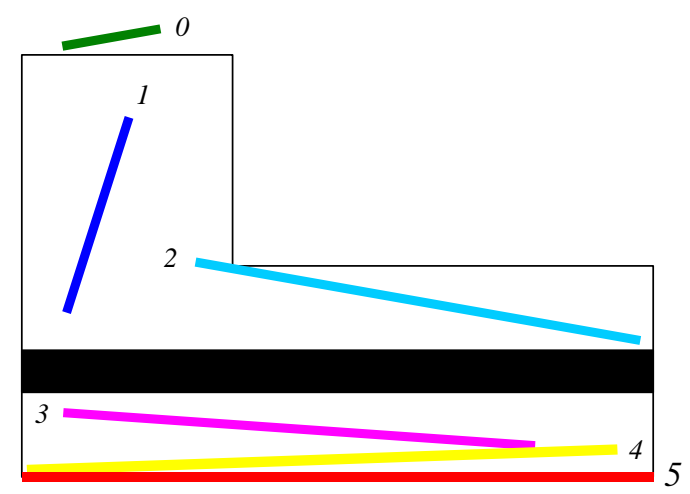

Fig. 22. Variation of stiffener layout during the optimization process (Case 3).

Case 3 is devised to show the ability of the proposed technique. The interior infeasible region is defined by a $2 \mathrm{D}$ planar convex polygon VI with four vertexes:

$$
\mathbf{V I}=\left[\begin{array}{l}
\mathbf{V} \mathbf{I}_{\mathbf{x}} \\
\mathbf{V} \mathbf{I}_{\mathbf{y}}
\end{array}\right]=\left[\begin{array}{llll}
0.0 & 1.5 & 1.5 & 0.0 \\
0.2 & 0.2 & 0.3 & 0.3
\end{array}\right] \quad \text { (unit: } \mathrm{m} \text { ) }
$$

The optimal stiffener layout of Case 3, marked number $\mathbf{5}$, is shown in Fig. 22, which is also nearly the same with the results of Cases 1 and 2 even though the feasible region is disjointed. The obtained feasible stiffener position is $\mathbf{x}^{*}=\left(x_{i}^{*}, y_{i}^{*}, L_{i}^{*}, \theta_{i}^{*}\right)=\left(0.0000 \mathrm{~m}, 0.0000 \mathrm{~m}, 1.5000 \mathrm{~m}, 0.0000^{\circ}\right)$ and the first natural frequency $\omega_{1}^{*}$ of the stiffened plate is $3.9967 \mathrm{~Hz}$, as shown in Fig. 23.

\subsection{Front panel of outdoor unit of air conditioner}

The objective of the second example is to raise the first flexible natural frequency of the front panel of an air conditioner outdoor unit by attaching a stiffener. Fig. 24 shows an FE model of the front panel, which is modeled by using MSC.PATRAN and imported into Structural Dynamics Toolbox. Four-node quadrilateral steel plate elements with thickness of $3 \mathrm{~mm}$ are used for the FE 


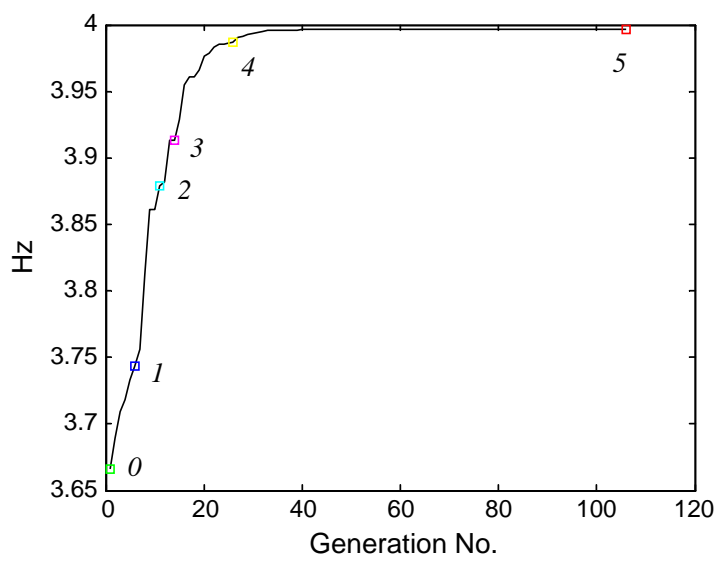

Fig. 23. Improvement of the first natural frequency of the stiffened plate (Case 3).

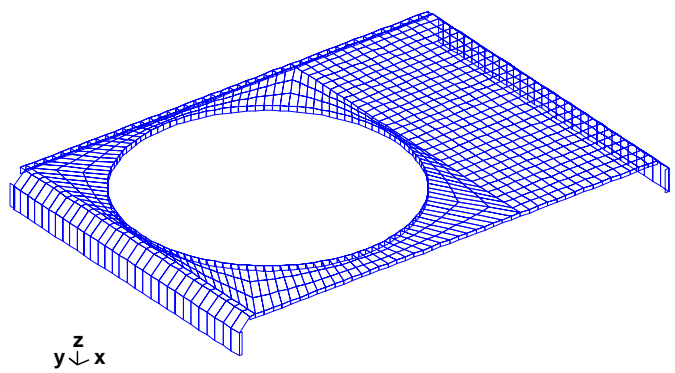

Fig. 24. FE model of front panel of air conditioner outdoor unit.

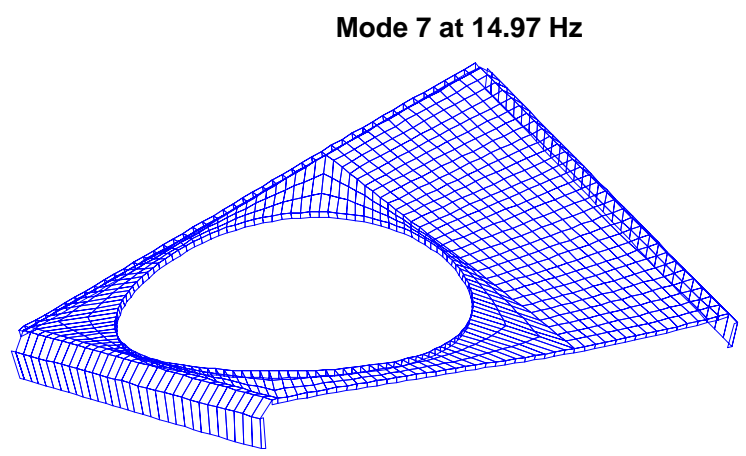

Fig. 25. First flexible mode shape of the front panel. 


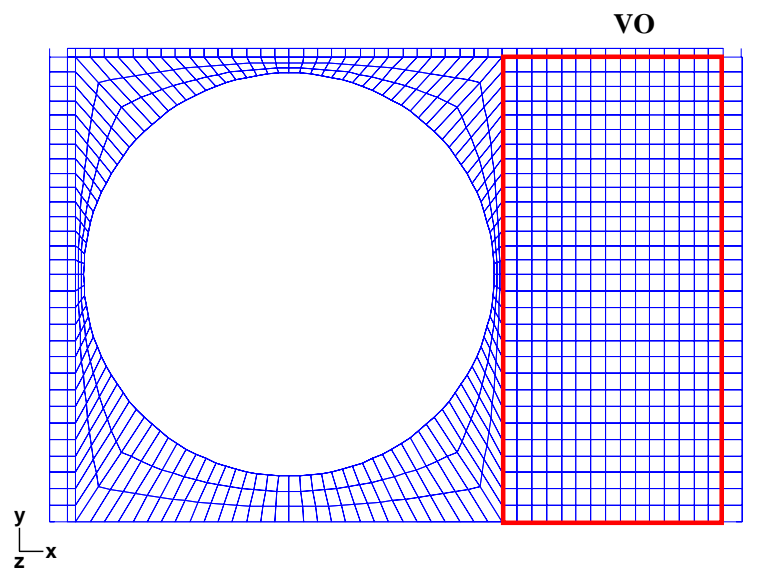

Fig. 26. Feasible region on the $z=0$ plane where a stiffener is placed.

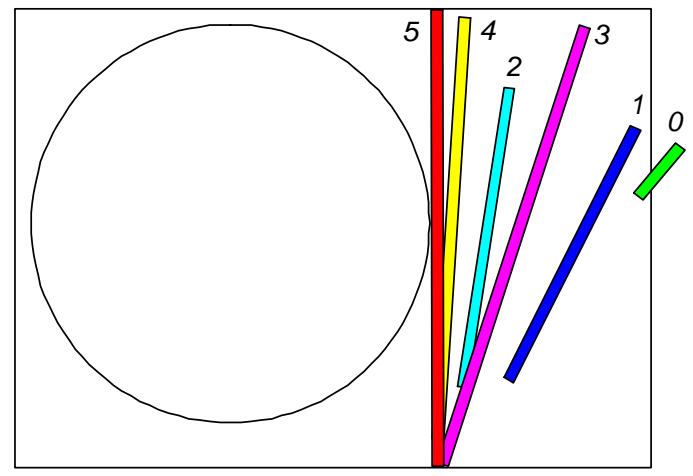

Fig. 27. Variation of stiffener layout during the optimization process of the front panel.

model. The total number of dofs is 8520 . First flexible mode $(14.9687 \mathrm{~Hz})$ is a torsional mode, as shown in Fig. 25. Assume that the stiffener is constrained to be placed within the specified feasible region in the front panel, as shown in Fig. 26. The feasible region is defined by a 2D planar convex polygon with four vertexes:

$$
\mathbf{V O}=\left[\begin{array}{l}
\mathbf{V} \mathbf{O}_{\mathbf{x}} \\
\mathbf{V O}_{\mathbf{y}}
\end{array}\right]=\left[\begin{array}{rrrr}
0.270 & 0.550 & 0.550 & 0.270 \\
-0.319 & -0.319 & 0.280 & 0.280
\end{array}\right] \quad \text { (unit: } \mathrm{m} \text { ) }
$$

The steel stiffener has cross-sectional dimensions of width $5 \mathrm{~mm}$ and height $10 \mathrm{~mm}$. Note that initial individuals are also positioned in infeasible region.

The obtained optimal stiffener layout is shown in Fig. 27, marked number 5. When the stiffener is placed vertically along the left boundary line of the feasible region, the first flexible natural frequency can be increased to $15.6088 \mathrm{~Hz}$, as shown in Fig. 28. 


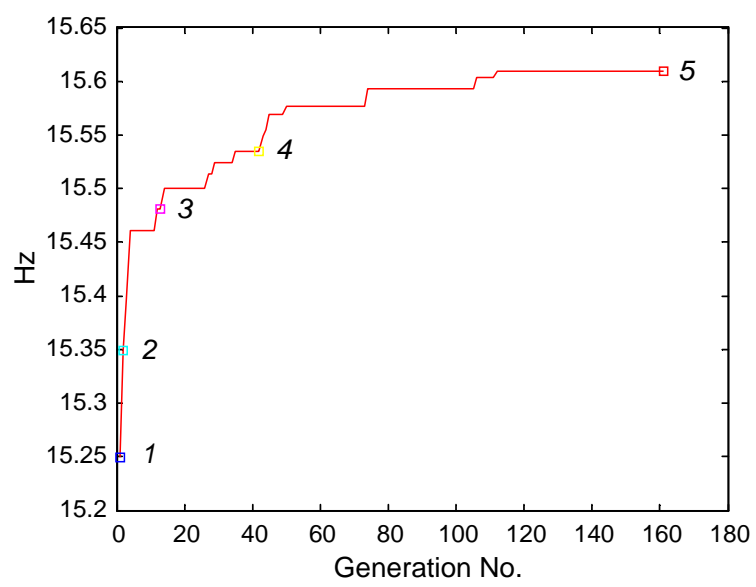

Fig. 28. Improvement of the first flexible natural frequency of the stiffened front panel.

\section{Conclusions}

In this paper, stiffener layout optimization to raise the first natural frequency of a base structure was performed, where the length as well as the position of the stiffener was chosen as a design variable. Evolution strategies was taken as an optimization tool. In addition, EVOSLINOC was utilized to handle both feasible and infeasible individuals. In EVOSLINOC, geometry algorithms were adopted to define both convex and concave feasible regions and to measure a degree of geometry constraint violation. From numerical examples, the proposed geometry constraint handling technique was verified and proved that the technique can easily be applied to structures in not only convex but also concave shapes, even with a protrusion or interior holes. Note that the proposed technique using geometry algorithms is not limited to stiffener layout optimization problems. The technique can also be applied to other research areas that are related to sound and vibration and need numerical optimization techniques for better designs such as absorptive material arrangement optimization, position optimization of supports, dampers and mounts, etc., where geometry constraints are inevitably involved.

\section{Acknowledgements}

This work was supported by a National Research Laboratory program (NRL:M1-0001-000139) financed by KISTEP (Korea Institute of Science and Technology Evaluation and Planning).

\section{References}

[1] P. Avitabile, Twenty years of structural dynamics modification - a review, in: Proceedings of the 20th International Modal Analysis Conference, Los Angeles, CA, USA, 2002, pp. 356-372.

[2] Y.H. Park, Y.S. Park, Structure optimization to enhance its natural frequencies based on measured frequency response functions, Journal of Sound and Vibration 229 (5) (2000) 1235-1255. 
[3] Z.S. Liu, J.S. Hansen, D.C.D. Oguamanam, Eigenvalue sensitivity of stiffened plates with respect to the location of stiffeners, Structural and Multidisciplinary Optimization 16 (1998) 155-161.

[4] M.A. Tournour, N. Atalla, Optimization design for stiffeners using component mode synthesis, in: Proceedings of Noise-Con 98, Ypsilanti, MI, USA, 1998, pp. 331-334.

[5] J.L. Marcelin, Genetic optimization of stiffened plates without the FE mesh support, International Journal for Numerical Methods in Engineering 54 (2002) 685-694.

[6] J. Fatemi, P. Trompette, Optimal design of stiffened plate structures, in: Proceedings of the 43rd AIAA/ASME/ ASCE/AHS/ASC Structures, Structural Dynamics, and Materials Conference, Denver, CO, USA, 2002, AIAA Paper 2002-1672.

[7] E.I. Jung, Y.S. Park, SDM based on substructures having non-matching nodes, in: Proceedings of International Conference on Structural Dynamics Modeling: Test, Analysis, Correlation and Validation, Funchal, Madeira, Portugal, 2002, pp. 655-662.

[8] E.I. Jung, Y.S. Park, Structure optimization using coupling position of substructure based on measured frequency response functions, in: Proceedings of the 32nd International Congress and Exposition on Noise Control Engineering (Inter-Noise 2003), Seogwipo, Jeju, Korea, 2003, pp. 3576-3583.

[9] J.H. Lee, Y.S. Park, SDM, utilizing evolution strategies: application to substructures having non-matching nodes, in: Proceedings of the 21st International Modal Analysis Conference, Kissimmee, FL, USA, 2003, Paper No. 114.

[10] J. O'Rourke, Computational Geometry in C, 2nd ed., Cambridge University Press, Cambridge, 1998.

[11] http://www.geometryalgorithms.com.

[12] M. Papadrakakis, N. Lagaros, G. Thierauf, J. Cai, Advanced solution methods in structural optimization based on evolution strategies, Engineering Computations 15 (1) (1998) 12-34.

[13] Z. Michalewicz, Genetic Algorithms + Data Structures = Evolution Programs, 3rd ed., Springer, Berlin, 1999.

[14] K. Ohkura, Y. Matsumura, K. Ueda, Robust evolution strategies, Applied Intelligence 15 (2001) 153-169.

[15] T.T. Binh, U. Korn, Scalar optimization with linear and nonlinear constraints using evolution strategies, Lecture Notes in Computer Science, vol. 1226, Springer, Berlin, 1997, pp. 381-392.

[16] C.A.C. Coello, Theoretical and numerical constraint-handling techniques used with evolutionary algorithms: a survey of the state of the art, Computer Methods in Applied Mechanics and Engineering 191 (2002) 1245-1287.

[17] K.C. Park, C.A. Fellipa, G. Rebel, A simple algorithm for localized construction of non-matching structural interfaces, International Journal for Numerical Methods in Engineering 53 (9) (2002) 2117-2142.

[18] E. Balmès, Structural Dynamics Toolbox Version 5.1 (for use with MATLAB), http://www.sdtools.com, October 2003. 